\title{
Giant compressive emphysema: a rare complication of COVID-19
}

\author{
Julien Rakotoson', Johary Andriamizaka Andriamamonjisoa $2^{*} \mathbb{C}$, Mandimbisoa Noely Oberlin Andriamihary ${ }^{5}$, \\ Solohery Jean Noël Ratsimbazafy ${ }^{3}$, Roger Dominique Randrianarimalala ${ }^{3}$, Rivo Andry Rakotoarivelo ${ }^{4}$ and \\ Stéphane Ralandison ${ }^{1}$
}

\begin{abstract}
Background: The severe acute respiratory syndrome coronavirus-2 (SARS-CoV-2) is a new ribonucleic acid (RNA) beta-coronavirus, responsible for a worldwide pandemic. Very few cases of SARS-COV-2-related emphysema have been described, except among patients with chronic obstructive pulmonary disease. The thoracic CT scan is the key examination for the diagnosis and allows to evaluate the severity of the pulmonary involvement. The prognosis of the patient with giant emphysema (GE) on coronavirus disease 2019 (COVID-19) in critical or severe form remains poor. We report an original case of COVID-19 pneumonia, critical form, complicated by a giant compressive left emphysema of $22.4 \mathrm{~cm}$ in a young subject without respiratory comorbidities.
\end{abstract}

Case presentation: A 34-year-old man was hospitalized for left laterothoracic pain. He had no prior medical history. The physical examination revealed tympany on percussion of the left lung. The CT scan confirmed COVID-19 pneumonia with 95\% lung involvement. Also, the presence of a voluminous left sub pleural emphysema of $22.4 \mathrm{~cm}$ with compression of the ipsilateral pulmonary parenchyma as well as the mediastinal structures towards the right side. The diagnosis COVID-19 pneumonia, critical form, complicated by a compressive left giant emphysema was made. He was put on oxygen, a dual antibiotic therapy, a corticotherapy, and curative doses of enoxaparin. A thoracic drainage surgery was performed at 24th day of hospitalization, which confirmed the giant emphysema. The patient remains on long-term oxygen therapy.

Conclusion: The COVID-19 has polymorphic manifestations, pneumonia is the most important one. There are relatively few reports associating COVID-19 and emphysema; furthermore, reports associating COVID-19 and giant emphysema are extremely scarce. CT scans can confirm the diagnosis and differentiate it from a pneumothorax. The pulmonary prognosis of the association of COVID-19 in its severe or critical form with giant emphysema remains poor.

Keywords: Emphysema, Giant, Coronavirus disease 2019, CT scan

\section{Background}

The severe acute respiratory syndrome coronavirus-2 (SARS-CoV-2) is a novel single-stranded ribonucleic acid (RNA) beta-coronavirus, which emerged in China in

\footnotetext{
*Correspondence: joharyandria24octobre@gmail.com

2 Department of Infectious Diseases, Faculty of Medicine Antananarivo,

Joseph Raseta Befelatanana University Hospital Antananarivo,

101 Antananarivo, Madagascar

Full list of author information is available at the end of the article
}

December 2019, responsible for the coronavirus disease 2019 (COVID-19), which is causing the current global pandemic [1]. Despite the polymorphism of this disease, pulmonary involvement remains the predominant presentation causing hypoxemic lung disease [2, 3]. Emphysema is the consequence of an irreversible destruction of the pulmonary parenchyma, with damage to the respiratory bronchiole and alveolar walls, causing an enlargement of the airways and responsible for chronic respiratory insufficiency. Giant emphysema (GE), on the 
other hand, causes acute respiratory failure and a severe compressive phenomenon. Since the pandemic, very few cases of SARS-COV-2 related emphysema have been described, except among patients with chronic obstructive pulmonary disease (COPD) [2,3]. Our aim is to report an original case of GE on SARS-CoV-2 lung disease, in a young subject without comorbidity.

\section{Case presentation}

A 34-year-old man was hospitalized in AntananarivoMadagascar for left laterothoracic pain associated with dyspnea. He reported no cigarette nor alcohol consumption, and denied any prior medical history including diabetes, hypertension, and respiratory diseases. He has no known respiratory history. There was no family history of alpha-1 antitrypsin deficiency, no notion of pneumothorax or spontaneous emphysema. He had been presenting for 10 days with headaches, diffuse arthromyalgia, and a hacking cough in a febrile context. Two days before his admission, the patient suffered from significant asthenia, followed by progressive dyspnea, chest pain aggravated by coughing and change of position.

The physical examination revealed pulsed oxygen saturation (SpO2) of $77 \%$, polypnea of 35 cycles per minute, blood pressure of $160 / 80 \mathrm{mmHg}$, heart rate of 117 beats per minute, temperature of $36.7^{\circ} \mathrm{C}$, and body mass index (BMI) of $23.1 \mathrm{~kg} / \mathrm{m}^{2}$. The patient was obnubilated, had difficulty speaking, with signs of acute respiratory distress, condensation syndrome of the entire right lung, auscultatory silence and tympany on percussion of the left lung. There was no subcutaneous snowy crepitus of the thoracic region. There was right deviation of regular heart sounds, turgidity of the jugular veins, without edema of the lower extremities or ascites.

Lab analyses made in the 2nd day of hospitalization showed a hyperleukocytosis of $27 \times 10^{3} \mathrm{k} / \mu \mathrm{L}$, including $96 \%\left(25 \times 10^{3} \mathrm{k} / \mu \mathrm{L}\right)$ neutrophils, $2 \%\left(0.54 \times 10^{3} \mathrm{k} / \mu \mathrm{L}\right)$ lymphocytes, a discrete thrombocytosis of $475 \times 10^{3} \mathrm{k} /$ $\mu \mathrm{L}$, and a C-reactive protein of $75 \mathrm{mg} / \mathrm{L}$. Renal and hepatic tests were unremarkable. D-dimer was elevated to $2571 \mathrm{ng} / \mathrm{mL}$. The nasopharyngeal COVID-19 polymerase chain reaction (PCR) performed on the 3rd day of hospitalization was positive. Human immunodeficiency virus (HIV) serology was negative. The chest X-ray at the patient's bed on the 2nd day of hospitalization showed an alveolar syndrome of the right lung with right deviation of the mediastinum and clarity of the right lung except at the apex (Fig. 1).

The diagnosis of COVID-19 was made, with a doubt on the complication: left emphysema or left pneumothorax. He was put on oxygen at $20 \mathrm{~L} / \mathrm{min}$ through a high-flow nasal cannula connected to an oxygen

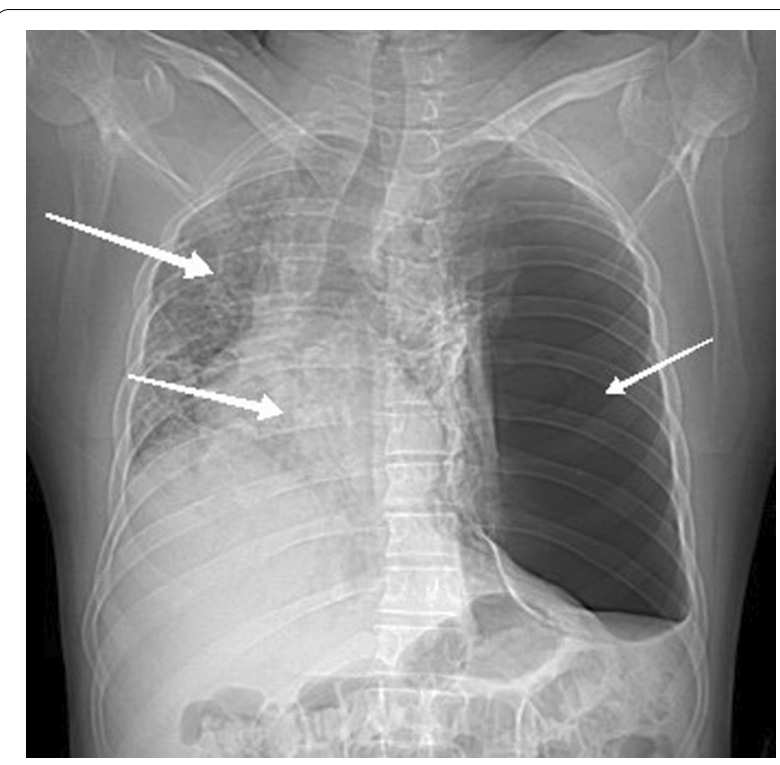

Fig. 1 Chest CT scan without contrast injection: the chest X-ray at the patient's bed showed an alveolar syndrome of the right lung (arrow: right/up) with right deviation of the mediastinum (arrow: right/down) and clarity of the right lung except at the apex (arrow: left)

cylinder; a dual antibiotic therapy with ceftriaxone slow direct intravenous $1 \mathrm{~g}$ daily for 10 days, associated with a roxythromycin $150 \mathrm{mg}$ tablet, twice daily for 10 days; a corticotherapy with dexamethasone intravenous direct: $24 \mathrm{mg}$ daily for 3 days, then $12 \mathrm{mg}$ daily for 7 days, then $8 \mathrm{mg}$ daily for 7 days, then $4 \mathrm{mg}$ daily for 6 day. A potassium supplementation with $600 \mathrm{mg} \times 3$ dose per day for 22 days; and curative doses of enoxaparin subcutaneous $0.6 \mathrm{ml}$ every $12 \mathrm{~h}$ for 10 days. The evolution was favorable with disappearance of signs of respiratory distress, with a $\mathrm{SpO} 2$ of $92 \%$ under $8 \mathrm{~L}$ of oxygen at 14th day of hospitalization.

The thoracic scanner was done late at 15 th day of hospitalization because of the dependence on oxygen therapy and the impossibility of moving the patient with a worsening of desaturation at the least movement. In addition, the radiology center is not on site, requiring a trip with an ambulance that has a capacity of $10 \mathrm{~L}$ of portable oxygen. The injection of contrast medium is not done because of the patient's lack of money. The result of the CT scan is in favor of a viral pneumonia on SARS-CoV-2 with pulmonary involvement of about $95 \%$ of the parenchyma, by the presence of ground glass opacities with multilobar and multisegmental internal reticulations. Also, the presence of a voluminous left sub pleural emphysema of $22.4 \mathrm{~cm}$ with compression of the ipsilateral pulmonary parenchyma as well as the mediastinal structures towards the right side. At the 
same time, we note the presence of some pulmonary emphysema bullae on the right side (Figs. 2, 3).

The diagnosis of COVID-19 pneumonia, critical form, complicated by a compressive left GE was made. Pneumothorax was ruled out by the presence of lung parenchyma at the apex and the left pulmonary base. Also, the absence of retraction of the left lung on the pulmonary hilum.

However, the tympanism of the left lung thorax and the deviation of the heart sounds persisted. On the 22nd day of hospitalization: the patient remained oxygenorequerent with $\mathrm{SpO} 2$ at $94 \%$ under $2 \mathrm{~L}$ of oxygen and desaturation at the slightest effort. A thoracic surgery was performed at 24th day of hospitalization, which confirmed the GE. The Surgery was done under general anesthesia with mechanical ventilation by a thoracotomy, then a bullectomy followed by placement of a chest tube. The chest tube was removed on day 6 of the operation. The operation was successfully performed to overcome the acute respiratory status. Nevertheless, the patient
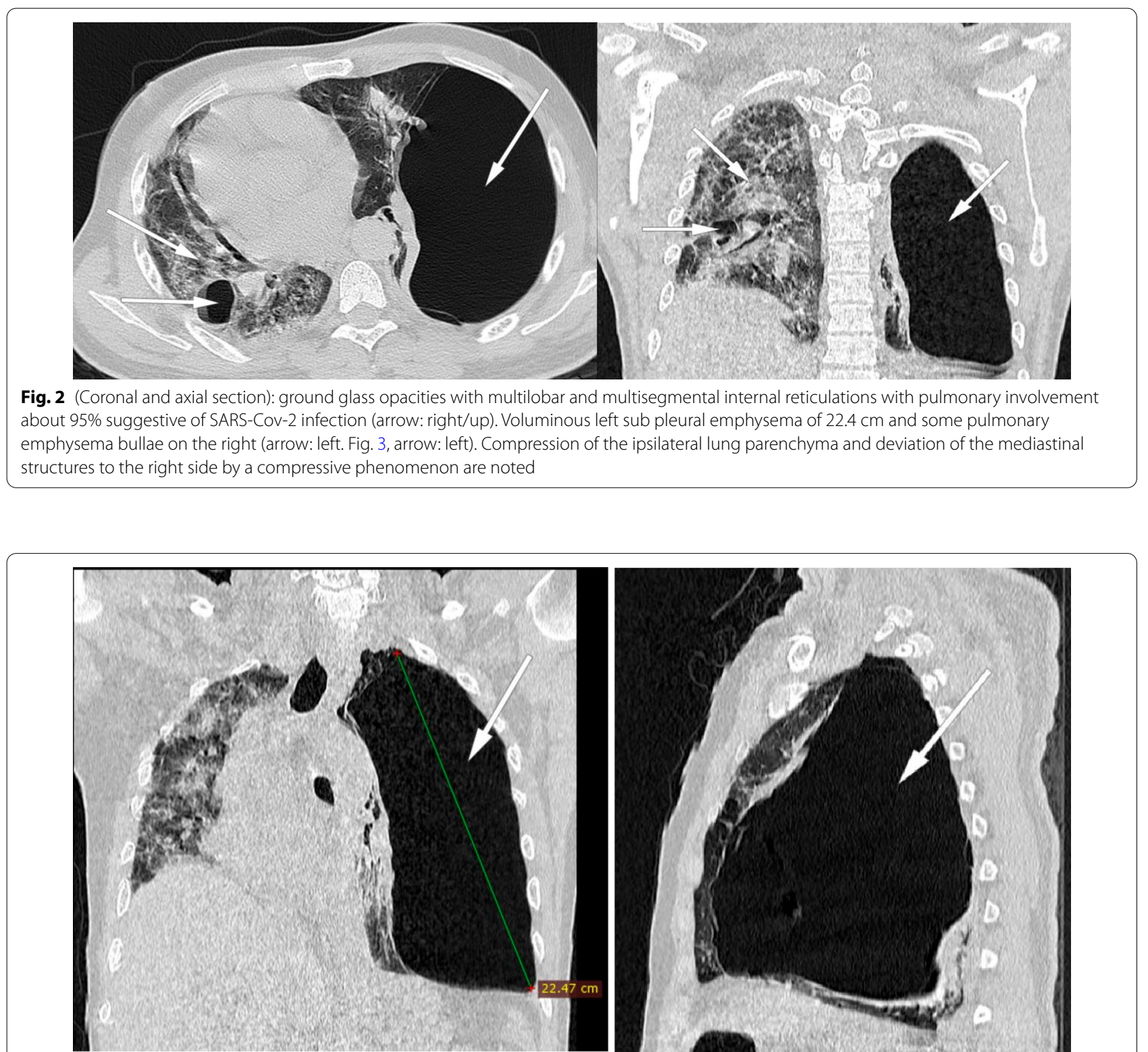

Fig. 3 (Coronal and sagittal section): ground glass opacities with multilobar and multisegmental internal reticulations with pulmonary involvement about 95\% suggestive of SARS-Cov-2 infection (Fig. 2, arrow: right/up). Voluminous left sub pleural emphysema of $22.4 \mathrm{~cm}$ and some pulmonary emphysema bullae on the right (Fig. 2, arrow: left. arrow: left). Compression of the ipsilateral lung parenchyma and deviation of the mediastinal structures to the right side by a compressive phenomenon are noted 
Table 1 Summarizing the clinical and laboratory characteristics of patient

\begin{tabular}{|c|c|}
\hline Chronology of the event & Clinical and biological characteristics of the patient \\
\hline 10th day before his hospitalization & Influenza-like syndrome, febrile context \\
\hline 2nd day before his hospitalization & Progressively worsening dyspnea and chest pain that are accentuated by coughing and changing position \\
\hline On admission & $\begin{array}{l}\text { Clinical characteristics: } \\
\text { No sign of shock } \\
\text { Acute respiratory distress syndrome with pulse oxygen saturation } 77 \% \text { on room air, polypnea at } 35 \text { cycles per } \\
\text { minute } \\
\text { Condensation syndrome of the entire right lung } \\
\text { Tympany and auscultatory silence of the left lung }\end{array}$ \\
\hline On admission & $\begin{array}{l}\text { Biological characteristics: } \\
\text { Hyperleukocytosis: } 27 \times 10^{3} \mathrm{k} / \mu \mathrm{L}, 96 \%\left(25 \times 10^{3} \mathrm{k} / \mu \mathrm{L}\right) \text { neutrophils, } 2 \%\left(0.54 \times 10^{3} \mathrm{k} / \mu \mathrm{L}\right) \text { lymphocytes } \\
\text { Thrombocytosis: } 475 \times 10^{3} \mathrm{k} / \mathrm{UL} \\
\text { C-reactive protein: } 75 \mathrm{mg} / \mathrm{L} \\
\text { D-dimer: } 2571 \mathrm{ng} / \mathrm{mL} \\
\text { The nasopharyngeal COVID-19 polymerase chain reaction (PCR): positive } \\
\text { HIV serology: negative }\end{array}$ \\
\hline 22nd day of hospitalization & $\begin{array}{l}\text { Disappearance of signs of respiratory distress } \\
\text { Pulse oxygen saturation } 94 \% \text { at } 2 \mathrm{~L} / \mathrm{min} \text { oxygen } \\
\text { Desaturation at the slightest effort }\end{array}$ \\
\hline 24th day of hospitalization & Bullectomy surgery, with thoracic drainage \\
\hline From the 7th day post-op & Long-term oxygen-dependent, at home \\
\hline 2 months after surgery & Died of a recurrence severe acute pneumonia \\
\hline
\end{tabular}

remains on long-term oxygen therapy by nasal cannula. The patient survived 2 months after his thoracic drainage, following a recurrence severe acute pneumonia.

\section{Discussion and conclusions}

In the absence of pathological lungs, COVID-19 can cause giant emphysema. The severity is related to the size of the emphysema, which is a source of compressive phenomenon. The CT scan is the key examination for diagnostic confirmation. The prognosis of the association remains poor.

Emphysema is a lung disease, characterized by the irreversible destruction of the respiratory bronchiole and the alveolar wall that lines the lungs. This leads to an enlargement of the distal airways with the formation of intra-parenchymal air bubbles [4]. It is purely related to COVID-19 if an infected patient has no history of chronic obstructive pulmonary disease or a history of familial alpha-1 antitrypsin deficiency. The association of emphysema with COVID-19 was less described in the first wave in 2020, but seems to be more numerous in the 2nd wave of 2021. Our case finds its originality in relation to the size of the emphysema, voluminous, measuring $22.4 \mathrm{~cm}$ long axis and its acquired character related to COVID-19. Also, because of the young age of our patient, non-smoker, which can eliminate the aggravation of preexisting emphysema or blebs ruptures.

The mechanism of our patient's GE may be different from that of Sun et al. Our patient had the signs of GE on admission (tympany with respiratory distress, and the image of emphysema in the chest X-ray). In other words, emphysema symptoms were the main reason for our patient's consultation. On the other hand, the case reported by Sun et al. presented the signs of emphysema during hospitalization where the patient had already received oxygen therapy at $20 \mathrm{~L} / \mathrm{min}$ [5]. COVID-19 itself and its treatment by excessive oxygen flow increase during desaturation could explain the occurrence of emphysema» [6]. The pathogenesis of emphysema primarily involves an inflammatory process in the lungs during various infections. Macrophages (CD 68+), neutrophils and CD8 $\mathrm{T}$ cells act on resident cells such as epithelial cells and fibroblasts via mediators (interleukin 8, TNF alpha, leukotriene) of inflammation, destroying the lung parenchyma. This mechanism occurs in the inflammatory phase of COVID-19 with alveolar-interstitial lung disease, in addition to the oxidative stress (formation of free radicals) created by the inflammatory process (2nd mechanism). The 3rd mechanism is an imbalance between pulmonary proteases-antiproteases often of genetic origin. The alpha 1 antitrypsin protein (protease inhibitor) plays a protective role. In case of its deficiency, this role is no longer ensured and the progressive destruction of the pre-existing alveolar walls causes the occurrence of emphysema [7]. This hypothesis is less likely in our case because there is no history of spontaneous pneumothorax in the family.

Any source of increased intra-thoracic pressure (exertion, coughing, constipation ...) can aggravate the size of an underlying emphysema or cause the rupture of a 
pre-existing bleb. Excessive ventilation can also aggravate or cause emphysema. Our patient has presented pulmonary tympany as soon as they admitted and had no invasive ventilation before the thoracic scanner. This eliminates the contribution of ventilation to the development of emphysema in this patient.

Critical CT involvement in COVID-19 is defined as $75 \%$ or more lung involvement. Our case presented up to $95 \%$ lung involvement, aggravated by the presence of a $\mathrm{GE}$ of $22.4 \mathrm{~cm}$. This association increases the risk of mortality of the disease $[7,8]$.

The CT scan is ideal imaging method for the diagnosis of emphysema and for the evaluation of its impact. It can differentiate between emphysema and pneumothorax or blebs. In case of COVID-19, chest CT has a sensitivity of $91.9 \%$ for the diagnosis, considered as a reference examination even in the early stage of the infection [9, 10]. It is therefore essential to perform a chest CT scan for any severe or critical form of COVID-19, to assess the severity of the lung involvement, to look for associated lesions, to have an idea of the prognosis and to provide a means of follow-up. Most COVID-19 related emphysemas described in the literature are small, with possible association with pneumomediastinum and pneumothorax. Regarding the therapeutic management, our patient was receiving oxygen therapy and surgery. The emphysema described in the literature was treated with oxygen therapy only [5]: non-invasive ventilation, high Flow Nasal Cannula and invasive mechanical ventilation [6]. In our case, surgery was necessary in view of the presence of signs of poor tolerance of the GE.

The association of COVID-19 and pulmonary emphysema is less described in the literature. Even more, GE is exceptional. It should be evoked in front of the asymmetry of the pulmonary examination with tympanism and the presence of signs of acute right heart failure. Only the CT scan can confirm the diagnosis and differentiate it from a pneumothorax. The pulmonary prognosis of the association of COVID-19 in its severe or critical form with GE remains poor (Table 1).

\section{Abbreviations \\ BMI: Body mass index; COPD: Chronic obstructive pulmonary disease; COVID 19: Coronavirus disease 2019; CT: Computerized tomography; GE: Giant emphysema; HIV: Human immunodeficiency virus; RNA: Ribonucleic acid; SARS-CoV-2: Severe acute respiratory syndrome coronavirus-2; SpO2: Pulsed oxygen saturation.}

\section{Acknowledgements}

We thank the team of the COVID-19 treatment center and the team of the thoracic surgery department of the JRB University Hospital, 101 Antananarivo-Madagascar

\section{Authors' contributions}

RJ, AAJ, AMNO: Draft of the introduction and observation of the clinical case. RSJN, RRD: Draft of the clinical case discussion. RAR, RS: Final correction and revision of bibliographic references. All authors read and approved the final manuscript.

Funding

No source of funding, self-financing.

Availability of data and materials

We cannot put the scanner online with the patient's information because of the confidentiality rule. On the other hand, imaging with patient information is available at the first author.

\section{Declarations}

Ethics approval and consent to participate

Not applicable.

\section{Consent for publication}

The first consent that was sent was signed by the patient's father, very early during the writing of the manuscript. The patient gave his verbal consent and asked his father to sign him because he was still very dysphenic and was not in a state of signed. On the other hand, now: the patient is already restored and goes much better. So we have reiterated his agreement directly and he agreed to signed. We had the patient's consent for the publication of the case and the CT images. Please find in PDF file separated written consent and signed by the patient. Note that patient and father have the same name.

\section{Competing interests}

The authors declare no conflict of interest.

\section{Author details}

${ }^{1}$ Department of Rheumatology, Faculty of Medicine Antananarivo, Morafeno University Hospital Toamasina, 501 Toamasina, Madagascar. ${ }^{2}$ Department of Infectious Diseases, Faculty of Medicine Antananarivo, Joseph Raseta Befelatanana University Hospital Antananarivo, 101 Antananarivo, Madagascar. ${ }^{3}$ Department of Internal Medicine, Faculty of Medicine Antananarivo, Military Hospital Soavinandriana Antananarivo, 101 Antananarivo, Madagascar. ${ }^{4}$ Department of Infectious Diseases, Faculty of Medicine Andrainjato Fianarantsoa, University Hospital Tambohobe Fianarantsoa, 301 Fianarantsoa, Madagascar. ${ }^{5}$ Department of Cardiology, Faculty of Medicine of Antananarivo, Joseph Rasta Befelatanana University Hospital, 101 Antananarivo, Madagascar.

Received: 8 June 2021 Accepted: 22 December 2021

Published online: 30 December 2021

\section{References}

1. Zhu N, Zhang D, Wang W, Li X, Yang B, Song J, et al. A novel coronavirus from patients with pneumonia in China, 2019. N Engl J Med. 2020;382(8):727-33.

2. Ma C, Wang X-L, Xie D-M, Li Y-D, Zheng Y-J, Zhang H-B, et al. Dynamic evaluation of lung involvement during coronavirus disease-2019 (COVID19) with quantitative lung CT. Emerg Radiol. 2020;27(6):671-8.

3. Ooi GC, Khong PL, Müller NL, Yiu WC, Zhou L, Ho JCM, et al. Severe acute respiratory syndrome: temporal lung changes at thin-section $C T$ in 30 patients. Radiology. 2004;230(3):836-44.

4. Uppaluri R, Mitsa T, Sonka M, Hoffman EA, McLennan G. Quantification of pulmonary emphysema from lung computed tomography images. Am J Respir Crit Care Med. 1997;156(1):248-54.

5. Sun R, Liu H, Wang X. Mediastinal emphysema, giant bulla, and pneumothorax developed during the course of COVID-19 pneumonia. Korean J Radiol. 2020;21(5):541-4.

6. Simioli F, Annunziata A, Polistina GE, Coppola A, Spirito VD, Fiorentino G. The role of high flow nasal cannula in COVID-19 associated pneumomediastinum and pneumothorax. Healthcare. 2021;9:620.

7. Sutherland ER, Martin RJ. Airway inflammation in chronic obstructive pulmonary disease: comparisons with asthma. J Allergy Clin Immunol. 2003:112(5):819-27.

8. Tabatabaei SMH, Rahimi H, Moghaddas F, Rajebi H. Predictive value of CT in the short-term mortality of Coronavirus Disease 2019 (COVID-19) 
pneumonia in nonelderly patients: A case-control study. Eur J Radiol. 2020;132:109298.

9. Hu Y, Zhan C, Chen C, Ai T, Xia L. Chest CT findings related to mortality of patients with COVID-19: A retrospective case-series study. PLOS ONE. 2020;15(8): e0237302. https://doi.org/10.1371/journal.pone.0237302.

10. Makhnevich A, Sinvani L, Cohen SL, Feldhamer KH, Zhang M, Lesser ML, et al. The clinical utility of chest radiography for identifying pneumonia: accounting for diagnostic uncertainty in radiology reports. AJR Am J Roentgenol. 2019;213(6):1207-12.

\section{Publisher's Note}

Springer Nature remains neutral with regard to jurisdictional claims in published maps and institutional affiliations.

- fast, convenient online submission

- thorough peer review by experienced researchers in your field

- rapid publication on acceptance

- support for research data, including large and complex data types

- gold Open Access which fosters wider collaboration and increased citations

- maximum visibility for your research: over $100 \mathrm{M}$ website views per year

At BMC, research is always in progress.

Learn more biomedcentral.com/submissions 